\title{
REVISTA
}

Edição Especial com publicação de trabalhos selecionados do V SIGEPRO

\section{APLICAÇÃO DA METODOLOGIA FMEA EM UM PROCESSO DE UMA INDÚSTRIA METALÚRGICA}

Bianca Michels Chaves ${ }^{1}$, Bruna Rigon de Oliveira ${ }^{*}$, Iasmim Elisa Teixeira ${ }^{1}$, Morgana Pizzolato ${ }^{1}$, Sarah Rabaioli ${ }^{1}$

${ }^{1}$ Departamento de Engenharia de Produção e Sistemas, Universidade Federal de Santa Maria, 97105-900, Santa Maria, Brasil.

*E-mail: brunarigon@live.com

\section{RESUMO}

No ambiente industrial, as empresas buscam criar vantagens competitivas em relação a seus concorrentes. Para isso, o processo produtivo deve ser acompanhado, a fim de identificar modos que o processo pode falhar e estabelecer ações corretivas para evitar que ocorram. Tendo isso em vista, o presente estudo tem como objetivo identificar os modos potenciais de falha de um processo em uma metalúrgica por meio da metodologia FMEA (Failure Mode and Effects Analysis). O processo escolhido como objeto deste estudo foi o corte de tarugos de aço, cuja análise verificou que as principais causas de falha são erros ligados a recursos humanos e que cerca de $30 \%$ dos modos potenciais de falhas geram paradas de produção. Assim, sugeriu-se a implementação de um plano de capacitação efetivo, bem como a execução de procedimentos de controle da qualidade, a fim de melhorar o desempenho do processo industrial em questão.

Palavras-chave: Manutenção Centrada em Confiabilidade (MCC). Análise de Modos de Falha e Efeitos (FMEA). Indústria Metalúrgica.

\section{Introdução}

O setor industrial é altamente competitivo e a busca pela melhoria contínua está cada vez mais presente na realidade das empresas, sendo que grandes produtores internacionais estão, cada vez mais, ganhando espaço no mercado nacional com produtos a preços mais competitivos do que os fabricados no Brasil [1]. Ademais, as inovações acontecem frequentemente, de forma que novos materiais, especificações e tecnologias surgem diariamente e, nesse ambiente em que as mudanças passaram a ser regra, as técnicas que auxiliam na identificação de falhas são ainda mais importantes [2].
Nesse cenário, a metodologia de Análise de Modos de Falha e Efeitos (FMEA) ganha destaque, auxiliando na busca por excelência em projetos e em processos. O método fornece subsídio para a identificação dos pontos fracos, contribuindo na busca da melhoria contínua [2]. Segundo Santos [3], a FMEA pode ser utilizada em diferentes áreas de uma organização, tais como: projetos de produtos, análise de processos, área industrial e/ou administrativa, manutenção de ativos e confiabilidade com o intuito de trazer importantes benefícios para o negócio.

Tendo em vista esse cenário, o problema central da pesquisa consiste na aplicação da metodologia FMEA em um processo de uma indústria metalúrgica situada na cidade de Santa 


\section{REVISTA}

Edição Especial com publicação de trabalhos selecionados do V SIGEPRO

Maria, localizada no estado do Rio Grande do Sul. Dessa forma, a importância do presente trabalho concentra-se em disseminar o conhecimento referente a metodologia em um ambiente fabril e complementar a literatura sobre o assunto.

Sendo assim, o objetivo geral desse trabalho é identificar os modos potenciais de falha no processo de corte de uma metalúrgica por meio da FMEA de processo. Para atender a esse objetivo geral, objetivos específicos devem ser atendidos, sendo eles: a) realizar o levantamento bibliográfico sobre o problema; b) estudar o processo produtivo da metalúrgica; c) aplicar os conhecimentos de FMEA no processo escolhido e d) propor soluções e planos de ação para a empresa.

\subsection{O elo entre a manutenção e a confiabilidade}

A Associação Brasileira de Normas Técnicas (ABNT) na NBR 5462 [4] define manutenção como uma combinação de ações técnicas e administrativas destinadas a manter ou recolocar um item em um estado no qual possa desempenhar uma função requerida. Zaions [5] complementa que as atividades de manutenção visam ainda a prevenção de falhas e a garantia da execução dos processos dentro dos parâmetros de disponibilidade, qualidade, prazos, custos e vida útil adequados. Além disso, segundo Slack [6], se a manutenção não consegue prevenir ou mesmo antecipar falhas, então os empenhos necessitam ser conduzidos para reduzir o impacto de tais falhas.

Consolidada no período da revolução industrial devido à necessidade de ajuste aos desgastes e quebras de equipamentos, a manutenção vem se aprimorando como uma estratégia para assegurar a melhoria contínua da qualidade de processos e produtos [5, 7]. Essa evolução deu origem ao que ficou conhecida como Manutenção Centrada em Confiabilidade (MCC). A MCC visa identificar os modos de falha que afetam as funções, determinar a importância de cada falha funcional a partir de seus modos de falha e selecionar as tarefas aplicáveis e efetivas na prevenção das falhas funcionais, visando garantir a confiabilidade e a segurança operacional dos equipamentos e instalações a um custo mínimo [8-9].

Lafraia [10] corrobora com ABNT [4], definindo que a análise da confiabilidade é a avaliação probabilística de um sistema funcionar dentro de limites estabelecidos, não falhando durante determinado período de tempo em certas condições ambientais, visando proporcionar um bom desempenho funcional com baixo índice de falhas de um produto. Dessa forma, para determinar a probabilidade de sobrevivência de um sistema em um determinado período, deve-se observar o comportamento da taxa de falhas, utilizando-se a modelagem dos tempos até a ocorrência da parada do equipamento [11].

A utilização de ferramentas como a análise de modos de falha e efeitos (FMEA) ou a análise da árvore de falhas (FTA) permite eliminar ou minimizar os modos de falha que são considerados críticos ao sistema, bem como criar uma sistemática de priorização de manutenção a custos baixos, com base na análise da sua confiabilidade $[10,12]$.

\subsection{Análise dos Modos e Efeitos Potenciais de Falha (FMEA)}

Segundo Rosa e Garrafa [13], a análise dos modos de falha e efeitos, denominada no meio industrial como FMEA, é um método de análise de projetos (de produtos ou processos, industriais e/ ou administrativos) usado para antecipar falhas que possam ocorrer em um produto, peça ou processo. Após a identificação das possíveis falhas, pode-se definir e tomar ações 


\section{REVISTA}

Edição Especial com publicação de trabalhos selecionados do V SIGEPRO

para reduzir ou eliminar a probabilidade de ocorrência ou, pelo menos, aumentar a probabilidade de detecção destas. Além disso, a ferramenta permite determinar o efeito dos problemas potenciais sobre o desempenho do sistema, mediante um raciocínio basicamente dedutivo [14].

O processo genérico para condução da FMEA constituise das seguintes etapas: descrever o produto ou processo; definir funções; descrever os potenciais modos de falha; descrever os efeitos de falhas; determinar as causas; definir métodos de controles ou controles atuais; calcular os riscos; planejar e implementar ações e avaliar os resultados [15].

Odlanicka-Poczobutt e Kulinska [16] ressaltam a importância da FMEA para o planejamento de medidas preventivas e a determinação da sua eficácia a partir da hierarquização de falhas e suas respectivas causas. A respeito disso, Gomes e Marinho [14] descrevem o método tradicional de priorização das causas de falhas potenciais a partir do cálculo do Índice de Risco, que é diretamente proporcional aos índices de Ocorrência, Detecção e Gravidade, sendo o produto dos três. Assim, quanto maior for o valor do risco em relação às diversas causas, maior deve ser a atenção dada para a resolução do problema. Os autores descrevem o Índice de Ocorrência $(\mathrm{O})$ como uma estimativa das probabilidades combinadas de ocorrer uma causa de falha; o Índice de Gravidade (G), como uma estimativa de quanto o efeito da falha impacta o cliente, assumindo que essa falha ocorra; e o Índice de Detecção (D), como a probabilidade de a falha ser detectada antes de chegar ao cliente.

A existência de dois tipos de FMEA, de Processo e de Produto (ou Projeto), é descrita por Luft et al. [17], sendo ambas constituídas pelas mesmas etapas e executadas de mesma maneira, diferenciando-se somente quanto ao objetivo. A FMEA de Produto considera as falhas que poderão ocorrer com o produto dentro das especificações do projeto. Já a FMEA de Processo leva em conta as possíveis falhas no planejamento e na execução do processo, também em relação às especificações do projeto. Para Aguiar e Mello [18], a utilização de FMEA de Processo elimina os pontos fracos do processo, reduzindo o risco de falhas a valores aceitáveis, objetivando a melhoria contínua e o registro histórico para futuros estudos.

Pantazopoulos e Tsinopoulos [19], Luft et al. [17], Odlanicka-Poczobutt e Kulinska [16] e Gomes e Marinho [14] relatam aplicações da ferramenta FMEA na indústria metalúrgica. Álvares, Souza e Fernandes [20], Fernandes, Matsuoka e Costa [21] e Leão e Andrade [22] analisam a utilização da FMEA para definição de estratégias de Manutenção Centrada em Confiabilidade, nos setores de geração de energia, construção civil e manufatura, respectivamente. Já Silva e Andrade [23] descrevem o uso de FMEA como subsídio para estabelecimento de atividades de manutenção em uma empresa do setor metal-mecânico.

\section{Metodologia}

O estudo foi realizado em uma metalúrgica localizada em um parque fabril da cidade de Santa Maria, no estado do Rio Grande do Sul, que presta serviços nas áreas de manutenção de máquinas, equipamentos industriais e agrícolas. A empresa trabalha com um portfólio de nove produtos diferentes, sendo referência na fabricação de roscas helicoidais.

A metodologia para aplicação da FMEA seguiu os passos de Fogliatto e Ribeiro [2], a qual possui treze etapas. Entretanto, para fins de simplificação, as etapas foram agrupadas em três como apresentado na sequência. 


\section{REVISTA}

Edição Especial com publicação de trabalhos selecionados do V SIGEPRO

- Etapa 1: Estudar a estratégia da empresa, escolher o processo e elaborar seu fluxograma;

- Etapa 2: Preencher a planilha da FMEA de processo definindo as seguintes variáveis: operação, os modos, efeitos e causas potenciais de falha, os controles de prevenção e detecção para, por fim, calcular o risco através das notas atribuídas às variáveis;

- Etapa 3: Recomendar ações para os itens de maior risco.

\section{Resultados e discussões}

Nessa seção, é apresentada a implementação da FMEA no processo de acordo com as etapas da metodologia.

A primeira etapa foi iniciada com um aprofundamento em relação a questões estratégicas da empresa. Foi observado que a estratégia competitiva dela é a velocidade de entrega ao cliente, com o objetivo de atender o mercado consumidor na fabricação de peças seriadas e na execução de projetos personalizados.

Para atender a essa estratégia, faz-se necessária a identificação das falhas que ocorrem nos principais processos da empresa. Dentre esses, o processo de corte possui grande importância para a qualidade final dos produtos entregues ao cliente, bem como pode contribuir com os atrasos à entrega em caso de falhas em seu processo. Dessa forma, o processo de corte, realizado com o auxílio da Serra Fita Franho 500-A, foi o escolhido para a realização do estudo.

Com a finalidade de visualizar as etapas do processo, foram estudados documentos pertinentes referentes ao processo de corte (manuais, procedimentos, histórico de falhas, etc.). Dessa forma, um fluxograma foi estruturado, onde fluxo se divide em quatro macro operações principais: desembarque da matériaprima, preparação, corte das peças e estocagem, e suas respectivas sub operações.

A operação de desembarque é iniciada com a compra das barras, na qual as especificações do tipo de material, comprimento e diâmetro são informadas ao fornecedor. Na sequência, o material é entregue ao centro fabril, ocorrendo a etapa de recebimento, na qual todas as barras são inspecionadas. Caso algo esteja fora do especificado, as barras são enviadas de volta para o fornecedor e um novo pedido é efetuado. Com as especificações corretas, o desembarque das barras é realizado e elas são armazenadas em prateleiras predefinidas, de acordo com o seu diâmetro.

Depois inicia-se a operação de preparação da matériaprima, constituído por diversas sub etapas. O processo é iniciado com o recebimento da ordem de fabricação (OF). Assim, o operador seleciona a barra conforme as especificações da OF e, na sequência, encontra o procedimento operacional que irá guiar o restante do processo. Após, o encarregado irá escolher a fita adequada para o tipo de barra, levantar a serra e posicionar a fita, fazer o ajuste da velocidade, realizar a medição da barra, ajustar o batente, posicionar a barra na máquina e baixar a serra, sempre levando em conta o procedimento operacional definido para tal. Por fim, antes de iniciar o processo de corte é realizado um teste de segurança e, em caso de falha, o processo é estagnado até a resolução do problema e a realização de um novo teste de segurança. Caso o teste seja satisfatório, o passo seguinte é o corte a barra.

Assim, inicia-se a operação de corte com o corte da barra e a medição da peça, com a finalidade de verificar se esta foi 


\section{REVISTA}

Edição Especial com publicação de trabalhos selecionados do V SIGEPRO

cortada conforme o tamanho especificado na OF. Caso a peça tenha dimensões inferiores às estabelecidas, esta é descartada do processo e encaminhada para reaproveitamento de material. No caso da peça ter sido cortada em um tamanho maior, esta é encaminhada para retrabalho.

A operação de estoque é iniciada no caso de a peça ter passado no teste de qualidade anterior, estando dentro do tamanho especificado. Nesses casos, a peça é estocada e posteriormente repassada para o próximo processo produtivo, não trabalhado no presente estudo.

Iniciando a etapa 2 da metodologia, após conhecer o processo de corte, fez-se necessário analisar detalhadamente o processo de corte com a Serra Fita Franho 500-A. Com o auxílio de um funcionário da fábrica, foram identificadas as operações envolvidas no corte com a serra e os possíveis eventos que caracterizam falhas em cumprir as funções do equipamento, sendo detectados 30 modos potenciais de falhas.

Com os modos de falhas e os efeitos identificados, determinou-se a Severidade para cada efeito listada através da identificação do impacto que o efeito tem na operação. A Severidade pode ser medida de 1 a 10, conforme escala estabelecida por Fogliatto e Ribeiro [2], onde 1 significa "efeito baixo" e 10 significa "efeito muito alto", seguindo a mesma lógica para as escalas intermediárias.

Na sequência, foram identificadas as possíveis causas para os modos potenciais de falha e as suas respectivas Ocorrências, as quais relacionam-se com a probabilidade da causa ocorrer. Foram encontradas 35 possíveis causas que foram medidas em uma escala de Ocorrência de 1 a 10, de acordo com
Fogliatto e Ribeiro [2], sendo 1 para "falhas muito improváveis" e 10 para "falhas quase inevitáveis", seguindo a mesma lógica para as escalas intermediárias. A definição contou com a ajuda de um funcionário da fábrica, que detém o conhecimento da dinâmica do chão de fábrica.

Além disso, os controles de prevenção foram identificados, os quais podem impedir as causas e os controles de detecção, os quais constatam a presença do modo de falha. Após o conhecimento dos controles dos processos já existentes, foram definidos os valores de Detecção, que se refere a uma estimativa da habilidade dos controles atuais detectarem causas ou modos potenciais de falhas. Usou-se a mesma escala anterior, de 1 a 10, onde 1 significa "probabilidade muito elevada de detecção" e 10 significa "probabilidade muito remota de detecção", com a mesma lógica para as classes intermediárias.

Após a mensuração dos valores de Severidade (S), Ocorrência $(\mathrm{O})$ e Detecção (D), torna-se possível obter o fator Risco (R), obtido através da equação abaixo, utilizado para priorizar ações de melhorias e correções.

$$
R=S * O * D
$$

A consolidação da etapa 2 deu-se com o preenchimento completo da planilha da FMEA. A Figura 1, a seguir, apresenta uma parte da planilha preenchida. 


\section{REVISTA}

\begin{tabular}{|c|c|c|c|c|c|c|c|c|c|}
\hline Operaçạio & Modo potencial de fallha & Efeito & $\mathrm{s}$ & Cassa & 0 & Controle de prevereçầo & Controle de deteccẵo & \begin{tabular}{|l|l}
$D$ & $R$ \\
\end{tabular} & \\
\hline Comprar barras & Comprar baras erradas & Parada de produçào & 5 & Desccuido do funcionàrio & 2 & $\begin{array}{l}\text { Treinamentolo dos } \\
\text { fincionirios }\end{array}$ & Planejamento do pedido & 20 & \\
\hline Receber bararas & Faltar barras & Paralad de produçąo & 5 & Atraso do fortecedor & 2 & $\begin{array}{l}\text { Banco de dados de } \\
\text { fomecedores }\end{array}$ & Inspeçà visual & \begin{tabular}{|l|l}
11 & 10
\end{tabular} & \\
\hline \multirow{3}{*}{$\begin{array}{l}\text { Inspecionarar as barasas no } \\
\text { recebimento }\end{array}$} & \multirow{2}{*}{$\begin{array}{c}\text { Aprover barras forta da } \\
\text { especificąạo }\end{array}$} & \multirow[t]{2}{*}{ Dificuldaded to corte } & 3 & $\begin{array}{c}\text { Procedimento de inspeção } \\
\text { mal tedigidio }\end{array}$ & 1 & w & $\begin{array}{l}\text { Rervisià gerencencial dos } \\
\text { procedimentos }\end{array}$ & \begin{tabular}{|l|l|}
2 & 6 \\
\end{tabular} & \\
\hline & & & 3 & Descuito do inspetor & 4 & Treinamento dos inspetores & Plano de amostragem & \begin{tabular}{|l|l|l|}
2 & 24 \\
\end{tabular} & \\
\hline & Fallar matéfia-prima & Paradad de produçàio & 6 & Falta de estoque da empresa & 2 & 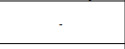 & $\begin{array}{c}\text { Plarailha de controle de } \\
\text { estoque }\end{array}$ & \begin{tabular}{|l|l|}
1 & 12 \\
\end{tabular} & 2 \\
\hline Desembarcar as baras & $\begin{array}{c}\text { Derrvbar baras duranteo } \\
\text { transpote }\end{array}$ & Dano do material & r. & Descuido do funcionâario & 2 & $\begin{array}{l}\text { Treinamentolo dos } \\
\text { funcionarios }\end{array}$ & Irspececa orisul & \begin{tabular}{|l|l|}
3 & 24 \\
\end{tabular} & \\
\hline $\begin{array}{c}\text { Alocara as barasas nas } \\
\text { prateletira comerspondente }\end{array}$ & $\begin{array}{l}\text { Alocar as baras na } \\
\text { prateleira inadequada }\end{array}$ & Dificuldade no corte & 2 & Descuido do funcionáaio & 4 & $\begin{array}{c}\begin{array}{c}\text { Treimanentoto dos } \\
\text { funcionarios }\end{array} \\
\end{array}$ & Inspeçąo visual & 16 & \\
\hline \multirow{2}{*}{$\begin{array}{l}\text { Receber ordem de } \\
\text { fabricaçâo( (OF) }\end{array}$} & \multirow{2}{*}{ 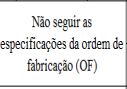 } & \multirow{2}{*}{\begin{tabular}{|c|}
$\begin{array}{c}\text { Retraballo ou descarte da } \\
\text { peca }\end{array}$ \\
$\begin{array}{c}\text { Retrablho oud descatte da } \\
\text { peeca }\end{array}$ \\
\end{tabular}} & 6 & Descuido do operador & 5 & $\begin{array}{l}\text { Treinamentios dos } \\
\text { operabores }\end{array}$ & Plano de anostragem & \begin{tabular}{|l|l}
2 & 60
\end{tabular} & \\
\hline & & & 6 & OF mal redipida & 2 & Patronizzçio das OFs & Plano de amostragem & $\begin{array}{lll}4 & 48 \\
\end{array}$ & \\
\hline $\begin{array}{c}\text { Escollera matéria prima } \\
\text { segrando a OF }\end{array}$ & \begin{tabular}{|c|}
$\begin{array}{c}\text { Seleçăo indequalata do } \\
\text { material }\end{array}$ \\
\end{tabular} & 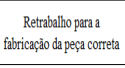 & 3 & Descuido do operador & 4 & 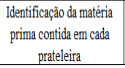 & Inspeçáa visistal & 36 & \\
\hline
\end{tabular}

Figura 1- Parte da planilha da FMEA para o processo de corte

Para recomendar ações (etapa 3), toda a planilha da FMEA foi analisada. Partindo do exemplo da Figura 1 é possível verificar que para a primeira operação, definida como "Comprar barras", pode ocorrer um modo potencial de falha, sendo ele a compra de barras erradas. Isso poderá gerar um efeito de parada de produção, que possui escala de Severidade 5, tendo como causa o descuido do funcionário, que possui uma Ocorrência de nível 2. Para que isso não ocorra, um controle de prevenção é o treinamento dos funcionários e um controle de detecção é o planejamento do pedido, que possuem escala de Detecção 2. Dessa forma, a operação de compra de barras possui um Risco totalizado em 20, podendo ser considerada uma operação de baixo risco em relação as demais. O mesmo procedimento foi realizado para as 19 operações identificadas para o processo estudado.

Considerando o conjunto de toda a FMEA, foi possível verificar que $30 \%$ dos modos potenciais de falhas geram paradas de produção, o que pode ser crítico para a dinâmica da fábrica, gerando paradas inesperadas. Esses modos são ocasionados principalmente por descuido dos funcionários, operadores e inspetores, causa que ocorreu 10 vezes na FMEA. Ademais, verificou-se que a maior parte das causas podem ser prevenidas por meio de treinamento dos funcionários, sendo um plano de ação a ser desenvolvido pela empresa. Por fim, foi constatado que o principal controle de detecção utilizado pela empresa é a inspeção visual, aparecendo 11 vezes, seguido pela supervisão e controle na segurança do trabalho.

Como última etapa do método utilizado, foram estruturadas ações para mitigação dos níveis de risco atrelados às principais causas de falhas, a partir da priorização de causas estabelecida pela FMEA. A priorização ocorreu com a utilização de um ponto de corte do nível de risco, que foi definido como $R=$ 60. A partir do ponto de corte, seis causas foram definidas como prioritárias na execução de ações de redução ou eliminação do risco. Obteve-se como principal causa de falha, com maior índice de risco $(R=140)$, o "Nível de óleo de refrigeração inadequado", que tem como modo potencial de falha a quebra da serra por superaquecimento e, como consequente efeito, uma parada de produção na operação de corte de matéria-prima.

As demais causas consideradas como principais têm como ponto em comum a ocorrência de erros humanos. Por exemplo, a causa "Uso incorreto do instrumento de medição pelo operador", $\operatorname{com} R=96$, durante a operação "Medir a peça", tem como modo potencial de falha a obtenção de dimensões fora dos limites de tolerância especificados na ordem de fabricação, ocasionando o corte incorreto do material. Outra causa relacionada a recursos humanos é a falta de conhecimento na operação "Corte da matéria-prima" $(R=84)$, verificada pelo modo potencial de falha da não imersão completa da bomba de refrigeração, tendo como efeito a danificação do equipamento.

Para essas causas, foram recomendadas ações de treinamento e auditoria das operações, a fim de atuar conforme o estabelecido no Manual de Procedimento Operacional dos 


\section{REVISTA}

Edição Especial com publicação de trabalhos selecionados do V SIGEPRO

equipamentos, já presente na empresa atualmente. Também foram sugeridas ações de valorização dos colaboradores, como oferta de bonificação aos funcionários com menor número de erros, por exemplo. A partir disso, verifica-se a importância de um programa de capacitação efetivo, bem como a aplicação de processos de controle da qualidade.

As causas priorizadas devido a seu maior índice de risco estão relacionadas a efeitos como parada da produção, corte incorreto de matéria-prima, danificação de equipamentos, acidentes de trabalho, retrabalho ou descarte de peças. Para evitálas, é recomendado o monitoramento permanente das variáveis Severidade, Ocorrência e Detecção, a fim de controlar os Riscos do processo e reavaliar a priorização de causas durante e após implementação das melhorias, para que novas ações de mitigação de risco possam ser identificadas e aplicadas.

A atuação do operador de máquina deve ser ressaltada na aplicação da ferramenta, visto que ele é quem tem contato rotineiro com o processo e conhece a fundo os modos potenciais de falha, suas causas e efeitos. Assim, o operador deve auxiliar no levantamento desses itens para que os resultados sejam validados, através de relatos e observações, para que a ferramenta seja atualizada ao longo do tempo.

A FMEA, aplicada ao processo de corte, permite que sua qualidade se mantenha em melhoria contínua, visto que visa a proposição e implementação de ações que mitiguem riscos ligados às causas de falhas. Dessa forma, com a diminuição dos índices de Ocorrência, Severidade e Detecção, há a redução da ocorrência de falhas e consequente aumento da confiabilidade do processo.

\section{Conclusões}

O presente estudo atendeu ao objetivo de identificar os modos potenciais de falha no processo de corte de uma metalúrgica por meio da FMEA de processo. Para isso, o processo foi estudado em detalhes para identificar os modos, os efeitos e as causas das falhas para, ao final, calcular os riscos a partir da Severidade, Ocorrência e Detecção, o qual tornou possível a recomendação de ações para mitigação dos maiores riscos.

Um total de 19 modos potenciais de falhas foram encontrados para o processo de corte, onde delimitou-se 33 efeitos e 33 causas possíveis para os modos de falhas. Além disso, a empresa já contava com controles de detecção, o qual atendiam a 28 efeitos. Ações foram recomendadas para os 6 maiores riscos resultantes da FMEA, a fim de reduzir ou cessar a ocorrência dos erros.

Além disso, visualizou-se que, uma parcela considerável dos modos potenciais de falhas, podem causar parada de produção, o que é bastante crítico para a empresa e que pode levar a diversos problemas para o chão de fábrica. Ademais, a causa mais recorrente para os modos de falhas foi o descuido dos funcionários, o que indicou uma possível ação de treinamento dos colaboradores envolvidos na operação de corte.

\section{FAILURE MODE AND EFFECTS ANALYSIS (FMEA) \\ APPLIED TO A METALLURGICAL INDUSTRY PROCESS}

ABSTRACT: Considering the industrial environment, companies seek to create competitive advantages over their fellow 


\section{REVISTA}

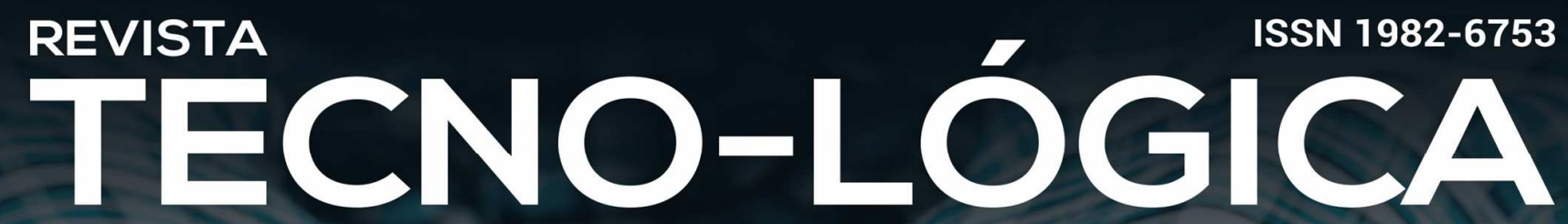

Edição Especial com publicação de trabalhos selecionados do V SIGEPRO

enterprises. With this in mind, the production processes should be monitored, in order to identify ways in which they may fail and to establish corrective actions to prevent these failures from occurring. The present study aims to identify the potential failure modes of a process in a metallurgical company, through the application of Failure Mode and Effects Analysis (FMEA). The process chosen as the object of this study is the cutting of steel billets, which analysis verified that its main causes of failure are related to human errors and about $30 \%$ of the potential failure modes generate production stops. Considering that, it is suggested the implementation of an effective employee training plan, as well as the execution of quality control procedures, in order to improve the performance of the industrial process in question.

Keywords: Reliability-Centered Maintenance (RCM). Failure Mode and Effects Analysis (FMEA). Metallurgical Industry.

\section{Referências}

[1] TRALESKI, A. V.; STEPIEN, H; BLAUTH, R. Q. Análise de modos de falha e seus efeitos no processo de produção de mancais. Revista de Gestão Industrial. v. 11, n. 02 , p. $70-86,2015$.

[2] FOGLiAtTO, F. S.; RIBEIRO, J. L. D. Confiabilidade e manutenção industrial. Rio de Janeiro: Elsevier, 2009.

[3] SANTOS, L. O. et al. Análise dos modos de falhas e seus efeitos (FMEA): uma avaliação das publicações em periódicos nacionais e internacionais. In: SIMPÓSIO DE ENGENHARIA DE PRODUÇÃO DE SERGIPE, 4., 2017, Sergipe. Anais...Sergipe: UFS, 2017.

[4] ASSOCIAÇÃO BRASILEIRA DE NORMAS TÉCNICAS (ABNT). NBR 5462: Confiabilidade e Mantenabilidade. Rio de Janeiro, 1994. 37 p.

[5] ZAIONS, D. R. Consolidação da Metodologia de Manutenção Centrada em Confiabilidade em uma Planta de Celulose e Papel. 2003. 219 p. Dissertação (Mestrado em Engenharia de Produção) - Universidade Federal do Rio Grande do Sul, Porto Alegre. 2003.

[6] SLACK, N.; CHAMBERS, S.; JOHNSTON, R. Administração da produção. 2. ed. São Paulo: Atlas, 2002.

[7] FÉLIX, J. C. et al. Proposição de melhorias em segurança por meio da aplicação do FMEA: um estudo de caso em uma empresa do setor ferroviário. Tecno-Lógica, v. 22, n. 2, p. 147-156, 2018.
[8] NUNES, E. L. Manutenção Centrada em Confiabilidade (MCC): análise da implementação em um sistema de manutenção consolidada. 2001. 146 p. Dissertação (Mestrado em Engenharia de Produção) - Universidade Federal de Santa Catarina, Florianópolis. 2001.

[9] MOUBRAY, J. Manutenção Centrada em Confiabilidade. São Paulo: Aladon Ltds, 2000, 426 p.

[10] LAFRAIA, J. R. B. Manual de Confiabilidade, Mantenabilidade e Disponibilidade. 2. ed. Rio de Janeiro: Qualitymark: Petrobrás, 2001.

[11] MACHADO, A.; ANDRADE, J.J.O. Emprego da confiabilidade para o estabelecimento de estratégias de manutenção na indústria metal-mecânica. In: ENCONTRO NACIONAL DE ENGENHARIA DE PRODUÇÃO, 33., 2013, Salvador. Anais... Salvador: ABEPRO, 2013.

[12] BRAILE, N. A.; ANDRADE, J. J. O. Estudo de falhas em equipamentos de costura industriais utilizando o FMEA e a análise de confiabilidade. In: ENCONTRO NACIONAL DE ENGENHARIA DE PRODUÇÃO, 33., 2013, Salvador. Anais... Salvador: ABEPRO, 2013.

[13] ROSA, L. C.; GARRAFA, M. Análise dos modos de falha e efeitos na otimização dos fatores de produção no cultivo agrícola: subprocesso colheita da canola. Gest. Prod., v. 16, n. 1, p. 63-73, 2009.

[14] GOMES, F. V.; MARINHO, M. H. N. Aplicação de FMEA e CEP para redução de sucata em uma indústria metalúrgica. Revista de Engenharia e Pesquisa Aplicada, v. 4, n. 3, p. 11-23, 2019.

[15] BACHEGA, S. J.; LIMA, A. D. Uso da Análise de Modo e Efeitos de Falha (FMEA) como apoio à redução do lead time do processo de orçamentação. In: ENCONTRO NACIONAL DE ENGENHARIA DE PRODUÇÃO, 30., 2010, São Carlos. Anais... São Carlos: ABEPRO, 2010.

[16] ODLANICKA-POCZOBUTT, M.; KULIŃSKA, E. The application of the FMEA method to failure analysis in the production process in a selected company of the metallurgical secondary manufacturing industry. In: METAL 2016 INTERNATIONAL CONFERENCE ON METALLURGY AND MATERIALS, 25., 2016, Brun, República Checa. Anais... Brun, República Checa: ICOM, 2016.

[17] LUFT et al. FMEA: aplicação no setor de engenharia em uma metalúrgica. In: SEMANA INTERNACIONAL DAS ENGENHARIAS DA FAHOR, 3., 2013, Horizontina. Anais... Horizontina: Faculdade Horizontina, 2013.

[18] AGUIAR, D. C.; MELlO, C. H. P. FMEA de Processo: uma proposta de aplicação baseada nos conceitos da ISO 9001:2000. In: ENCONTRO NACIONAL DE ENGENHARIA DE PRODUÇÃO, 28., 2008, Rio de Janeiro. Anais... Rio de Janeiro: ABEPRO, 2008.

[19] PANTAZOPOULOS, G.; TSINOPOULOS, G. Process Failure Modes and Effects Analysis (PFMEA): A Structured Approach for Quality Improvement in the Metal Forming Industry. Journal of Failure Analysis and Prevention, [S.I.], v. 5, n. 2, p. 5-10, 2005.

[20] ÁLVARES, A. J.; SOUZA, R. Q., FERNANDES, L. P. Análise FMEA Para Aplicação da Metodologia de Manutenção Centrada em Confiabilidade: Estudo de Caso em Turbinas Hidráulicas. In: CAIP - CONGRESSO INTERAMERICANO DE COMPUTAÇÃO APLICADA À INDÚSTRIA DE PROCESSOS, 8., 2007, Assunção, Paraguai. Anais... Assunção, Paraguai: CAIP, 2007. 


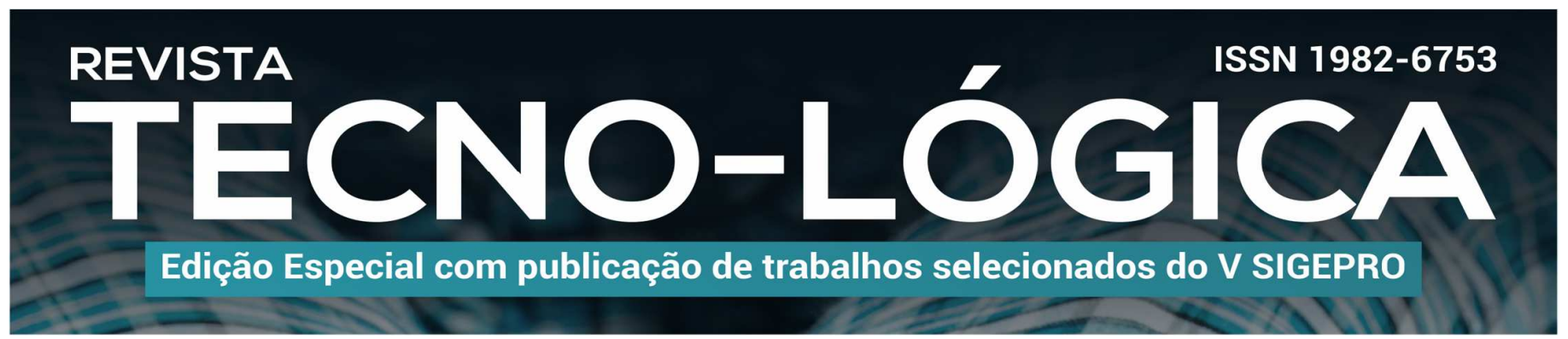

[21] FERNANDES, C. E. N.; MATSUOKA, J. V.; COSTA, E. S. Avaliação do sistema de gestão de qualidade utilizando o MCC - Manutenção Centrada na Confiabilidade na análise de falhas da construção civil. GETEC, v. 7, n. 19, p. 7292, 2018.

[22] LEÃO, N. S. M. S; ANDRADE, J. J. O. Aplicação da FMEA e análise de falhas em um equipamento de trafilagem para estabelecimento de estratégias de manutenção: estudo de caso. Revista Espacios, v. 36, n. 8, p. 1, 2015.

[23] SILVA, F. G.; ANDRADE, J. J. O. Análise de falhas de equipamentos da indústria metal-mecânica como subsídio para estabelecimento de atividades de manutenção. Revista Espacios, v. 37, n. 12, p. 17, 2016. 